\title{
Baseline Chronic Kidney Disease and Ischemic Method of Partial Nephrectomy Are Important Factors for the Short- and Long-Term Deterioration in Renal Function for Renal Cell Carcinoma Staged T1-T2: A Retrospective Single Center Study
}

\author{
Sung Han Kim, Jae Young Joung, Ho Kyung Seo, Kang Hyun Lee, and Jinsoo Chung \\ Department of Urology, Center for Prostate Cancer, Research Institute and Hospital of the National Cancer Center, \\ Goyang, Republic of Korea \\ Correspondence should be addressed to Jinsoo Chung; cjs5225@ncc.re.kr
}

Received 21 October 2016; Accepted 28 November 2016

Academic Editor: Péter Nyirády

Copyright (C) 2016 Sung Han Kim et al. This is an open access article distributed under the Creative Commons Attribution License, which permits unrestricted use, distribution, and reproduction in any medium, provided the original work is properly cited.

\begin{abstract}
The renal functions of 215 patients ( 24 with benign renal mass, the rest with RCC staged T1-T2) who underwent partial nephrectomy (PN) between 2003 and 2014 were evaluated to identify predictors of short- and long-term deterioration in renal function after PN among renal cell carcinoma (RCC) patients with or without preoperative predisposition to chronic kidney disease (CKD) and among patients with benign renal mass. The 1- and 5-year predictive factors for de novo CKD were statistically analyzed. The incidence of de novo CKD differed significantly $(p<0.001)$ among patients with benign renal mass, those with RCC but no preoperative CKD predisposition, and those with RCC combined with preoperative CKD predisposition. Independent predictors for de novo CKD at 1 year postoperatively included intraoperative ischemic method, ECOG score, elevated albumin levels, male sex, and smoking exposure (in pack-years). Predictors for de novo CKD at 5 years postoperatively included hypertension, high preoperative albumin levels, De Ritis ratio (aspartate aminotransferase/alanine aminotransferase ratio), smoking exposure, and preoperative predisposition to CKD. Preoperative predisposition to CKD and ischemic method applied during PN, along with other preoperative parameters, were important factors affecting postoperative renal function deterioration in patients with T1-T2 RCC.
\end{abstract}

\section{Introduction}

With the improvement in diagnostic modalities and widespread implementation of early screening systems, the ability to detect small, early-stage, localized renal cell carcinoma (RCC) has increased, which has made it possible to initiate oncological treatment with improved safety and efficacy, resulting in prolonged survival for patients with RCC [1]. Furthermore, the standard treatment strategy for RCC staged T1-T2 has changed from radical nephrectomy to partial nephrectomy (PN), taking into account comorbidities and life expectancy [2], as a strong association was found between renal function decline and the surgical management of small renal masses [2-4]. PN should also be recommended as the first therapeutic option for other types of RCC including familial RCC, von Hippel-Lindau RCC, or bilateral RCC, as well as in patients with a history of underlying chronic renal disease.

The effect of various aggravating and protective factors was investigated during the postoperative follow-up of RCC patients who underwent $\mathrm{PN}$, with the aim to identify the key aspects involved in preserving renal function and preventing or delaying the development of chronic kidney disease (CKD) [5]. It was found that the volume of resected renal tissue, which affects postoperative renal function, depends on the location and size of the tumor lesions, the presence of peritumoral structures, and renal vascular state. However, it is not easy to predict the postoperative development of CKD in RCC patients because that would require monitoring very many factors during the follow-up, whose range of action may or may not overlap in time (i.e., some should be monitored during the short-term follow-up, while others during the 
long-term follow-up). Moreover, the moment and direction of aggravation of renal function in RCC patients were only evaluated in comparison to the evolution of patients who underwent PN but had normal kidneys.

Several groups have attempted to describe the functional outcomes after renal surgery in terms of serum creatinine levels as indicative of kidney function $[6,7]$. However, the National Kidney Foundation guidelines assert that the assessment of renal function should be based on the estimated glomerular filtration rate (eGFR), which is a more accurate measure of kidney function because it considers serum creatinine levels in addition to the patient's age, sex, race, and body mass index. A reduced eGFR has been associated with increased risk of death, cardiovascular events, and hospitalization [8]; end-stage renal disease, characterized by low eGFR, leads to significant morbidity and mortality and represents a major burden to any healthcare system.

Therefore, in the present study, we monitored the change in renal function, assessed in terms of eGFR, in patients who underwent PN for RCC staged T1-T2. The patients were stratified into two groups based on their predisposition to $\mathrm{CKD}$, and these two groups were compared against a control group (which included patients who underwent PN for benign renal mass) in terms of renal function. The development of de novo CKD and the predictive factors for renal deterioration were evaluated statistically for the first and fifth year after PN in patients with a predisposition for CKD and in those without such predisposition.

\section{Patients and Methods}

2.1. Ethical Statement. Following approval by the Institutional Review Board (IRB) of the Research Institute and Hospital of the National Cancer Center (IRB approval number: NCC2014-0193), every patient record was anonymized and deidentified prior to analysis. All study protocols were conducted according to the ethical guidelines of the World Medical Association Declaration of Helsinki-Ethical Principles for Medical Research Involving Human Subjects. The need for written consent was waived by the IRB.

\subsection{Patients. The Kidney Cancer Database of the National} Cancer Center in Korea was searched for records of RCC patients who underwent PN between 2003 and 2014. The medical records of 252 RCC patients who underwent PN in that period were identified in order to retrospectively evaluate the development of de novo CKD and its predictive factors. Patients with a single kidney, metastatic RCC, other metastases to the kidney, or any preoperative histories of kidney intervention or therapies were excluded, resulting in a final enrollment of 215 patients including 24 (11.2\%) PN patients with benign renal mass. The cases with benign renal masses diagnosed pathologically after $\mathrm{PN}$ included 6 renal cysts, 1 pyelonephrosis, 1 pheochromocytomas, 3 cystic nephromas, 9 angiomyolymphomas, 1 hemangioendothelioma, 1 Castleman's disease, 1 mucinous adenoma, and 1 case with no tumor. All enrolled patients had complete follow-up data regarding renal function for at least one year postoperatively, whereas $124(57.7 \%)$ patients had such data for five years postoperatively.

The clinicopathological parameters including intraoperative findings, smoking history, and laboratory findings were used for the analysis of predictive factors for renal functional deterioration within one year and after five years from PN. Renal function was assessed based on serum creatinine levels evaluated at the following points: preoperatively and at $1,3,6,9,12,24,36,48$, and 60 months postoperatively. Preexisting CKD was defined as a preoperative eGFR < $60 \mathrm{~mL} / \mathrm{min} / 1.73 \mathrm{~m}^{2}$ (stage 3 according to the National Kidney Foundation guidelines) [9]. Postoperative CKD, acute kidney injury, and renal functional deterioration were defined either as a decline in the renal function, using the classification given in the National Kidney Foundation guidelines, from stages 1 or 2 to stage 3 , or as a decline in the renal condition from stage $3 \mathrm{~A}\left(\mathrm{eGFR}, 45-59 \mathrm{~mL} / \mathrm{min} / 1.73 \mathrm{~m}^{2}\right)$ to stage $3 \mathrm{~B}$ (eGFR, $30-44 \mathrm{~mL} / \mathrm{min} / 1.73 \mathrm{~m}^{2}$ ) [9].

2.3. Definition of Groups by Preoperative Condition. Two groups of RCC patients were defined according to the preoperative state of the renal function: the preoperative nonCKD RCC group (non-CKD group) and the preoperative CKD RCC group (CKD group). A third group was defined including the patients with benign renal disease (control group).

\subsection{Definition of Renal Function Aggravation for Each Group.} Renal function deterioration was defined differently for each group. For the non-CKD and control groups, it was defined as having a postoperative $\mathrm{eGFR}<60 \mathrm{~mL} / \mathrm{min} / 1.73 \mathrm{~m}^{2}$; for the CKD group, it was defined as a decline in the renal function from $\mathrm{CKD}$ stage $3 \mathrm{~A}$ to $3 \mathrm{~B}$ or from $\mathrm{CKD}$ stage $3 \mathrm{~B}$ to 4 [9].

2.5. Statistical Analyses. The differences in the occurrence of postoperative de novo $\mathrm{CKD}$ or renal functional deterioration at the first year and at the fifth year of follow-up were statistically evaluated for the three groups. Time to progression either to renal functional deterioration or to de novo CKD development was assessed using the Kaplan-Meier analysis with the log-rank test. The predictive risk factors for renal functional deterioration or de novo CKD development were analyzed using the Cox-regression hazard analysis with backward selection (alpha $=0.05)$. Statistical analyses were performed by using Stata software (Release 9.2, StataCorp, College Station, TX, USA). A $p$ value of $<0.5$ was deemed statistically significant.

\section{Results}

A total of $145(67.4 \%)$ patients were included in the nonCKD group, whereas 46 (21.4\%) patients were included in the CKD group (Table 1). The median follow-up time for all patients was 43 months (12-134 months). All RCC patients (88.8\%) had T1 stage RCC and only $0.5 \%$ experienced tumor recurrence despite the tumor-free resection margin. A total of $84.6 \%$ of the patients underwent open $\mathrm{PN}$, with a median ischemic time of 21 minutes; warm ischemia was applied in 
TAble 1: Patient baseline demographics $(N=215)$.

\begin{tabular}{|c|c|c|}
\hline Parameter & Median (range) & Percentage or SD \\
\hline Age (years) & $55(24-78)$ & \\
\hline Gender (male/female) & $154 / 61$ & $71.6 / 28.4$ \\
\hline $\operatorname{BMI}\left(\mathrm{kg} / \mathrm{m}^{2}\right)$ & $24.7(16.6-39.8)$ & \\
\hline \multicolumn{3}{|l|}{ Underlying disease } \\
\hline Diabetes & 32 & 14.9 \\
\hline Hypertension & 89 & 41.4 \\
\hline Hypercholesterolemia & 9 & 4.2 \\
\hline Ischemic heart disease & 5 & 2.3 \\
\hline Anticoagulation therapy history & 8 & 3.7 \\
\hline Aspirin therapy & 21 & 9.8 \\
\hline ASA score $0 / 1 / 2$ & $112 / 95 / 8$ & $52.1 / 44.2 / 3.7$ \\
\hline ECOG 0/1/2 & $143 / 70 / 2$ & $66.5 / 32.6 / 0.9$ \\
\hline Smoking & 121 & 56.3 \\
\hline Smoking volume (PY) & $8(0-87)$ & \\
\hline Follow-up duration (mo) & $43(12-134)$ & \\
\hline \multicolumn{3}{|l|}{ Preoperative laboratory findings } \\
\hline Hemoglobin & $14(8.6-17.5)$ & \\
\hline Albumin & $4.6(3.6-5.9)$ & \\
\hline Calcium & $9.4(8.3-10.9)$ & \\
\hline Total Cholesterol & $185.5(97-335)$ & \\
\hline De Ritis ratio (AST/APT) & $1.1(0.3-3.1)$ & \\
\hline Creatinine & $1.0(0.6-1.8)$ & \\
\hline Estimated GFR & $69.7(37.3-103.4)$ & \\
\hline Preoperative PADUA score & $8.0(5-12)$ & \\
\hline Tumor number & $2.4(0.3-16)$ & \\
\hline Low/intermediate/high & $67 / 86 / 62$ & $31.2 / 40.0 / 28.8$ \\
\hline Longitudinal: Inf/interpolar $/ \mathrm{med} / \mathrm{mid} / \mathrm{sup}$ & $60 / 42 / 3 / 60 / 50$ & 27.9/19.5/1.4/27.9/23.3 \\
\hline RENAL score & $7(3-18)$ & \\
\hline Ant/post & $107 / 108$ & $49.8 / 50.2$ \\
\hline \multicolumn{3}{|l|}{ Disease category } \\
\hline \multicolumn{3}{|l|}{ Preoperative } \\
\hline CKD RCC & 46 & 21.4 \\
\hline Non-CKD RCC & 145 & 67.4 \\
\hline Benign renal mass & 24 & 11.2 \\
\hline \multicolumn{3}{|l|}{ Operative method } \\
\hline Laparoscopy & 33 & 15.4 \\
\hline Open & 182 & 84.6 \\
\hline \multicolumn{3}{|l|}{ Pathologic T stage } \\
\hline $\mathrm{T} 1$ & 193 & 89.8 \\
\hline $\mathrm{T} 2$ & 22 & 10.2 \\
\hline \multicolumn{3}{|l|}{ Fuhrman grade } \\
\hline 1 & 20 & 9.3 \\
\hline 2 & 131 & 60.9 \\
\hline 3 & 43 & 20.0 \\
\hline 4 & 2 & 1.4 \\
\hline Unknown & 19 & 8.8 \\
\hline Margin positive & 30 & 14.0 \\
\hline Safety resection margin (mm) & $2.0(1.0-9.0)$ & \\
\hline Ischemic method warm/cold/no & $131 / 42 / 42$ & $60.9 / 19.5 / 19.5$ \\
\hline Ischemic time (min) & $21(0-70)$ & \\
\hline Number of clamping vessels & $2(1-4)$ & \\
\hline Tumor diameter $(\mathrm{cm})$ & $2.0(0.5-2.2)$ & \\
\hline
\end{tabular}


TABLE 1: Continued.

\begin{tabular}{lcr}
\hline Parameter & Median (range) & Percentage or SD \\
\hline Postoperative 5-year follow-up & $1.6(0.6-2.2)$ & $78.1(29-118.8)$ \\
$\quad$ Creatinine & & 77.7 \\
$\quad$ eGFR & 167 & 7.4 \\
Histology & 16 & 3.7 \\
$\quad$ Clear cell, pure & 8 & 11.2 \\
Papillary & 24 & 0.5 \\
Chromophobe & 1 & $52.1(4.8-48.8)$ \\
$\quad$ Benign renal mass & & \\
Recurrence & & \\
Time to recurrence
\end{tabular}

SD, standard deviation; MSKCC, Memorial Sloane Kettering Cancer Center; LN, lymph node; F/U, follow-up; PD, progressive disease; SD, stable disease; PR, partial response; CR, complete response; RECIST, Response Evaluation Criteria In Solid Tumors.

TABLE 2: Creatinine and estimated GFR data at baseline $(N=215)$ and postoperative first $(N=215)$ and last year $(N=124)$.

\begin{tabular}{lc}
\hline & $\begin{array}{c}\text { Median (min-max, } \\
\text { range) }\end{array}$ \\
& or $($ percentage, \\
\hline Baseline & $1.0(0.6-1.8)$ \\
Baseline sCr & $69.7(37.3-103.4)$ \\
Baseline eGFR & \\
First year & $2.0(1-4)$ \\
Change of sCr at first year & $69.5(25.6-129.1)$ \\
Change of eGFR at first year & $14(6.5)$ \\
First year CKD & $13(2-16)$ \\
Time to CKD at first year (mo.) & $32(14.9)$ \\
Fifth year & $35.4(0.9-133.7)$ \\
Last CKD & $1.0(0.6-2.2)$ \\
Time to CKD at fifth year & $78.1(29-118.8)$ \\
Change of sCr at fifth year & \\
Change of eGFR at fifth year &
\end{tabular}

most cases (60.9\%). Other baseline demographics including clinicopathological characteristics and intraoperative findings are described in Table 1.

When comparing the data regarding renal function changes from the 5-year follow-up with those from the 1year follow-up, there were higher changes in eGFR after 5years than after 1 year $\left(5\right.$ years, $78.1 \mathrm{~mL} / \mathrm{min} / 1.73 \mathrm{~m}^{2} ; 1$ year, $69.5 \mathrm{~mL} / \mathrm{min} / 1.73 \mathrm{~m}^{2}$ ) and higher development of de novo CKD after 5 years than the after 1 year ( 5 years, $14.9 \%$; 1 year, $6.5 \%$; Table 2). The incidence of de novo CKD in the control, non-CKD, and CKD groups was, respectively, $0 \%, 2.8 \%$, and $5.2 \%$ after 1 year and $0 \%, 3.2 \%$, and $6.5 \%$ after 5 years (both $p<0.001$, Figure 1). However, the difference regarding the incidence of de novo CKD between the control and the nonCKD groups was not statistically significant after 1 year $(p=$ 0.648 ); compared to the control group, the non-CKD group had a decline in renal function after five years of follow-up (Figure 1(b)). The median time to develop CKD, as identified after 1 and 5 years after PN, was 13 (2-16) months and 35.4 $(0.9-133.7)$ months, respectively $(p<0.001$; Table 2 , Figure 1$)$.

The results of the multivariate analyses of predictive factors for renal functional deterioration or de novo CKD development, given in terms of hazard ratio (HR) and 95\% confidence interval $(95 \% \mathrm{CI})$, for each variable that showed significance at 1 year after PN $(p<0.05$, Table 3$)$ were as follows: cold ischemia, $\mathrm{HR}=0.053,95 \% \mathrm{CI}=0.004-$ 0.699; no ischemia, HR $=0.077,95 \% \mathrm{CI}=0.007-0.827$; Eastern Cooperative Oncology Group (ECOG) score 1, HR $=0.0002,95 \% \mathrm{CI}=0.0001-0.077$; ECOG score $0, \mathrm{HR}=$ $0.002,95 \% \mathrm{CI}=0.0001-0.203$; preoperative albumin levels, $\mathrm{HR}=0.010,95 \% \mathrm{CI}=0.0001-0793$; male sex, $\mathrm{HR}=31.401$, $95 \% \mathrm{CI}=3.037-324.649$; and smoking exposure expressed as pack-years, $\mathrm{HR}=1.061,95 \% \mathrm{CI}=1.021-1.1036$. For each variable that showed significance at 5 years after PN $(p<$ 0.05 , Table 3 ), the results of the multivariate analyses were as follows: hypertension, $\mathrm{HR}=16.991,95 \% \mathrm{CI}=2.666-108.298$; preoperative albumin levels, $\mathrm{HR}=28.172,95 \% \mathrm{CI}=2.177-$ 364.577; De Retis ratio of aspartate aminotransferase/alanine aminotransferase (AST/ALT), HR $=13.772,95 \% \mathrm{CI}=1.330$ 142.550; smoking exposure, $\mathrm{HR}=1.081,95 \% \mathrm{CI}=1.028-1.137$; and preoperative $\mathrm{CKD}, \mathrm{HR}=13.158,95 \% \mathrm{CI}=1.654-104.659$.

\section{Discussion}

In the present study, we evaluated renal functional deterioration and its predictive factors at one and five years after PN in patients with RCC staged T1-T2. The CKD and non-CKD groups accounted for $21.4 \%$ and $67.4 \%$ of our study sample, respectively, which is in agreement with previously reported rates for CKD (20-24\%) [9]. The RCC patients were stratified into $\mathrm{CKD}$ or non-CKD groups according to the preoperative state of their renal function, to rule out the oncologic effect of RCC itself on the change in renal function and to differentiate among the factors predisposing for CKD in RCC patients whose renal function is recovering after PN. In addition, we also considered a control group, which included patients who underwent PN for removal of benign renal masses. We examined and compared the postoperative changes in renal 

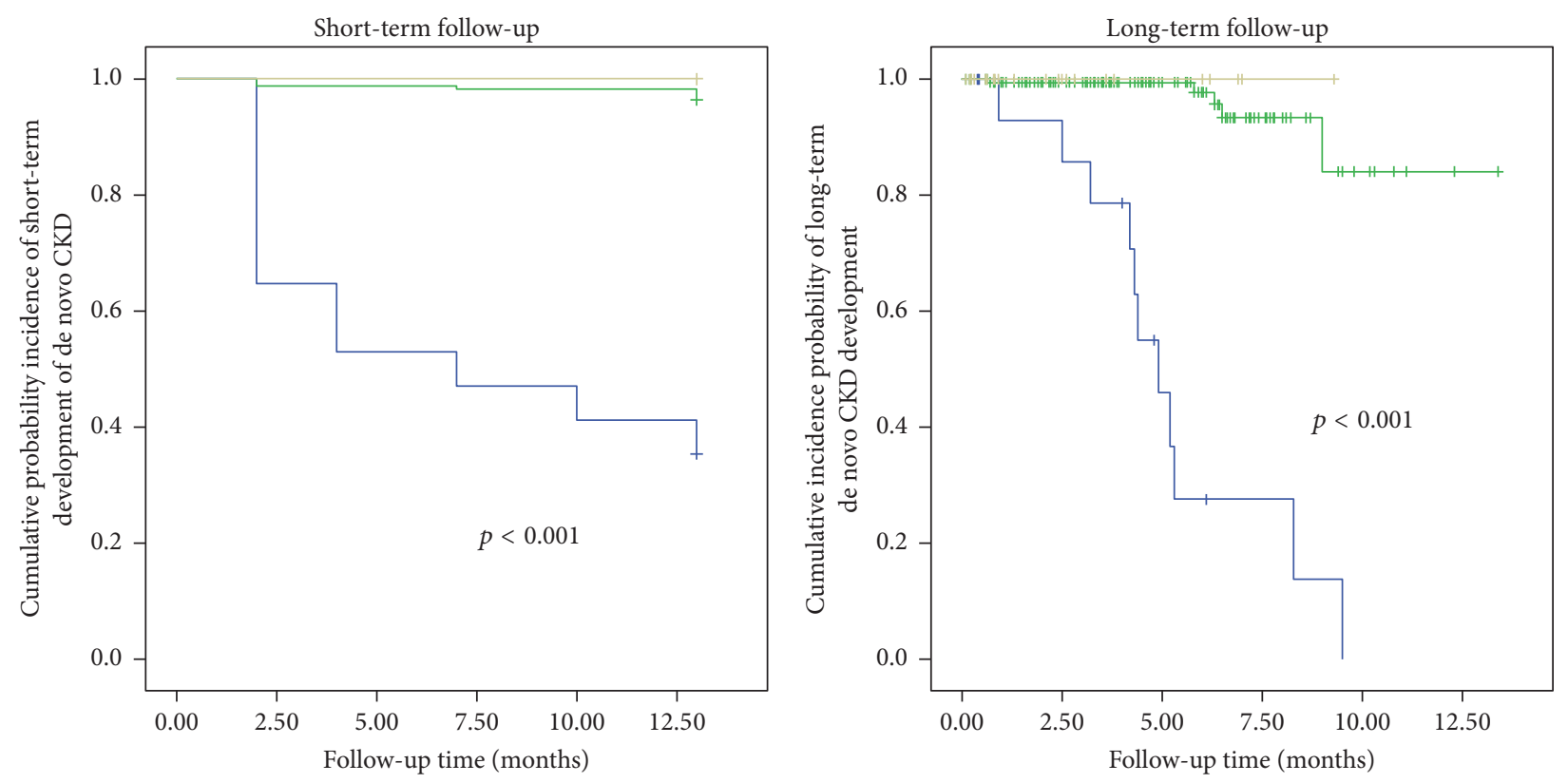

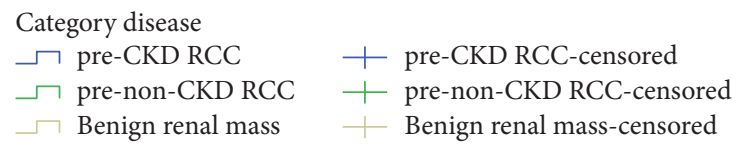

(a)

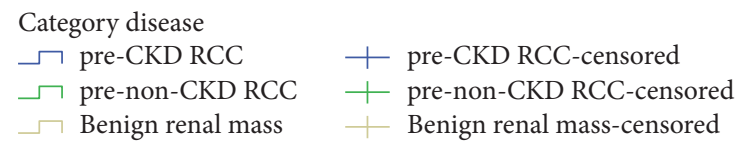

(b)

FIGURE 1: Incidence curve for chronic kidney disease (CKD) (a) during the short-term (first year postoperatively) and (b) during the longterm (fifth year postoperatively) follow-up in patients with renal cell carcinoma (RCC) staged T1-T2, treated with partial nephrectomy.

function for patients of the CKD, non-CKD, and control groups.

As expected, the changes in renal function indicated more deterioration and higher incidence of de novo CKD within 5 years after PN than within the first year (14.9\% versus $6.5 \%$; Table 2). The number of patients who developed de novo CKD was almost twice as high $(p<0.001$; Figure 1$)$ in the CKD group than in the non-CKD group, both during the first year (5.2\% versus $2.8 \%$ ) and during the fifth year $(6.5 \%$ versus $3.2 \%$ ) of follow-up, whereas none of the patients in the control group showed postoperative development of CKD. Furthermore, for the first postoperative year, there was no statistically significant difference between the control group and the non-CKD group regarding the number of patients with de novo CKD (Figure 1(b)). These results indicate that, in patients with small localized RCC staged T1-T2, the longterm deterioration of renal function is influenced mostly by the baseline state of the renal function rather than by the PN procedure or by RCC itself. Therefore, an active, close monitoring of renal function is necessary in such high-risk patients undergoing $\mathrm{PN}$, especially those already predisposed to $\mathrm{CKD}$ in the preoperative stage. Patients with benign tumors undergoing PN are unlikely to experience deterioration of the renal function merely due to PN itself. However, the renal function in RCC patients with no preoperative CKD should be monitored for at least 5 years after PN, and further investigation should be performed to identify and monitor potential aggravating factors.
We assessed the differences between short-term- and long-term-acting risk factors for renal function deterioration and development of de novo CKD. We considered all known risk factors reported in previous studies [4, 8-13]. Only smoking exposure was found as a significant factor for both the short-term and long-term deterioration of renal function ( $p<0.05$; Table 3). Smoking is known to have detrimental effects on the state of the vessels and kidneys, and heavier smoking may have a more significant effect on renal function recovery $[1,7]$.

We found that the intraoperative ischemic method (cold ischemia, $\mathrm{HR}=0.053$; no ischemia, $\mathrm{HR}=0.077$ ) represented a significant preventive factor for development of de novo CKD in the short term, which is similar to what has been reported in previous studies $[12,14]$. The ischemic method prevented ischemic changes in the nephrons. The no-clamping technique without ischemia was the best PN procedure for preservation of renal function, where possible. However, this observation might be affected by the fact that most of the renal masses included in our study were staged $\mathrm{T} 1(89.8 \%)$ and represented small-sized $(2.0 \mathrm{~cm})$ RCC. Under such circumstances, the removal of a small volume of kidney tissue is not expected to critically influence the degree of postoperative deterioration in renal function. In addition, our multivariate analysis did not indicate any significance of ischemic time in terms of CKD prediction $(p>0.05)$, despite the fact that univariate analysis showed that ischemic time differed significantly among the groups $(29.5 \pm 13.0 \mathrm{~min}$ for 
TABLE 3: Multivariate analysis of predictive risk factors with backward selection $(p=0.05)$ for 1-year short-term and 5-year long-term renal functional deterioration.

\begin{tabular}{|c|c|c|c|c|}
\hline & \multirow{2}{*}{$p$ value } & \multirow{2}{*}{ Hazard ratio } & \multicolumn{2}{|c|}{$95.0 \%$ confidence interval } \\
\hline & & & Lower & Upper \\
\hline \multicolumn{5}{|l|}{ Significant parameters at 1 year } \\
\hline Sex & 0.004 & 31.401 & 3.037 & 324.649 \\
\hline Smoking volume & 0.003 & 1.061 & 1.021 & 1.103 \\
\hline Albumin & 0.039 & .010 & .0001 & .793 \\
\hline Hemoglobin & 0.077 & 1.619 & .949 & 2.765 \\
\hline \multicolumn{5}{|l|}{ Ischemic method } \\
\hline Warm & 0.076 & & & \\
\hline Cold & 0.026 & .053 & .004 & .699 \\
\hline None & 0.034 & .077 & .007 & .827 \\
\hline \multicolumn{5}{|l|}{ ECOG } \\
\hline Group 2 & 0.012 & & & \\
\hline Group 1 & 0.004 & .0002 & .0001 & .077 \\
\hline Group 0 & 0.009 & .002 & .0001 & .203 \\
\hline \multicolumn{5}{|l|}{ Significant parameters at 5 years } \\
\hline Hypertension & 0.003 & 16.991 & 2.666 & 108.298 \\
\hline Preoperative Albumin (mg/dl) & 0.011 & 28.172 & 2.177 & 364.577 \\
\hline Calcium level (mg/dl) & 0.132 & .135 & .010 & 1.829 \\
\hline De Ritis ratio & 0.028 & 13.772 & 1.330 & 142.550 \\
\hline ASA class 1 & 0.001 & & & \\
\hline ASA class 2 & 0.317 & .101 & .001 & 8.968 \\
\hline ASA class 3 & 0.212 & 16.464 & .202 & 1343.216 \\
\hline Smoking volume (PY) & 0.003 & 1.081 & 1.028 & 1.137 \\
\hline Predisposing CKD & 0.015 & 13.158 & 1.654 & 104.659 \\
\hline
\end{tabular}

ECOG, Eastern Cooperative Oncology Group score; De Ritis ratio, AST/APT; ASA, American Society of Anesthesiologist Score; CKD, chronic kidney disease.

the CKD RCC group, $21.9 \pm 21 \mathrm{~min}$ for the non-CKD RCC group, and $10.7 \pm 16.1 \mathrm{~min}$ for the control group; $p<0.001$; data not shown). A recent systematic review showed that warm ischemia does not harm long-term renal function as long as ischemia time is kept between 20 and 25 minutes [15].

We also found that, in the short term, further significant factors influencing the postoperative evolution of renal function were male sex (HR, 31.401), albumin levels (HR, 0.010), and ECOG score $(0$ and $1, \mathrm{HR}<1.0)$, which were previously reported as factors affecting renal function [16-18]. Patients with good nutritional status and general performance in the preoperative stage are expected to have good baseline renal function and postoperative recovery after PN [16]. Compared to female patients, male Korean patients have a higher tendency of exposing themselves to negative social factors that may affect the recovery of renal function, likely because of their stressful occupational environment and habit of social drinking $[17,19,20]$. Another interesting finding regarding short-term risk factors was that an ECOG score of 1 was associated with less deterioration of renal function than an ECOG score of 0 , probably because patients with ECOG score 1 were more likely to visit their physician for health issues and have their renal function closely monitored by clinicians, who intervened sufficiently early to prevent or delay renal function deterioration or detect newly developed, small renal masses.

As for the long-term factors, we found no protecting factors but did identify detrimental factors such as hypertension $(\mathrm{HR}=16.991)$, high levels of serum albumin $(\mathrm{HR}=28.172)$, high De Ritis ratio $(\mathrm{HR}=13.772)$, and baseline predisposition to $\mathrm{CKD}(\mathrm{HR}=13.158)$ in addition to smoking exposure (HR $=1.081$ ). These findings are in line with those reported by previous studies $[1,7,9,10,17,20-22]$. In particular, hypertension and predisposition for CKD represent well-known aggravating factors in combination with smoking exposure, causing systemic cardiovascular changes and deterioration in renal function [10, 17, 20-22]. Interestingly, our multivariate analysis did not indicate diabetes as a significant predictor of short- or long-term CKD development, in spite of the significant differences in the incidence of diabetes among the three groups (3.7\% in the CKD RCC group, 9.8\% in the nonCKD RCC group, and $1.4 \%$ in the control group; $p=0.002$; data not shown). This observation is likely related to the fact that our study sample included patients with RCC staged T1$\mathrm{T} 2$, which required removing only a small volume of tissue during PN; furthermore, most patients did not exhibit severe 
diabetic state, suggesting that this aspect did not significantly affect kidney function and, consequently, was not related to the development of CKD postoperatively.

Abnormalities in albumin levels and De Ritis ratio values are factors indicating chronic liver disease [23]. It is well known that patients with chronic liver disease also had poor renal function, mostly related to the development of acute kidney injury, precipitated by either an acute disturbance of hemodynamics or an acute structural damage to the kidneys [10]. The incidence of chronic renal failure has been rising, due to increasing prevalence of conditions such as diabetes or viral hepatitis, which can be associated with renal damage. In addition, AST and ALT levels, which provide the De Ritis ratio [24], have been previously reported as significant prognostic biomarkers in several malignancies including kidney diseases [25]. The mechanism underlying the relationship of AST and RCC is related to the vital role of AST in glycolysis by relocation of NADH into the mitochondria through the malate-aspartate shuttle pathway, in which clear cell RCC with VHL loss was known to induce the expression of hypoxia factors known to be connected to extensively increased glycolysis in the mitochondria, which is well-known as the Warburg effect [26, 27]. No studies have ever suggested the importance of De Ritis ratio on postoperative renal functional changes after renal surgeries for which the levels of hepatic factors and serum albumin are significant.

Our study has several limitations related to the small sample size and retrospective design. Not all the patients completed at least five years of follow-up, and thus the longterm evaluation of renal function and underlying hepatic diseases was incomplete for such patients. However, it is clinically relevant to identify significant risk factors (among which those related to hepatic disease) for renal function deterioration, and our study is the first to identify longterm-acting risk factors related to hepatic function that affect postoperative renal function in RCC patients who underwent PN. Further prospective studies with long-term follow-up are warranted to evaluate the role of hepatic function in the recovery of renal function.

\section{Conclusion}

The study showed the significant predisposal of CKD patients for long-term renal function deterioration, and the significant influence of intraoperative ischemic method and time for short-term renal function deterioration in patients with T1T2 RCC who underwent PN. Further parameters including smoking exposure, hypertension, preoperative albumin levels, and De Ritis ratio values were also significant factors for postoperative renal functional deterioration. Careful patient selection for postoperative general management, as well as intraoperative planning, may help reduce this unfavorable outcome in renal function.

\section{Competing Interests}

The authors declared no competing interests.

\section{Authors' Contributions}

Sung Han Kim, Jae Young Joung, Ho Kyung Seo, Kang Hyun Lee, and Jinsoo Chung contributed to project development, data collection, and manuscript writing. Jae Young Joung, Ho Kyung Seo, Kang Hyun Lee, and Jinsoo Chung assisted in data collection. Sung Han Kim helped in manuscript writing.

\section{References}

[1] S. Weikert and B. Ljungberg, "Contemporary epidemiology of renal cell carcinoma: perspectives of primary prevention," World Journal of Urology, vol. 28, no. 3, pp. 247-252, 2010.

[2] R. H. Thompson, S. A. Boorjian, C. M. Lohse et al., "Radical nephrectomy for pTla renal masses may be associated with decreased overall survival compared with partial nephrectomy," The Journal of Urology, vol. 179, no. 2, pp. 468-472, 2008.

[3] W. C. Huang, E. B. Elkin, A. S. Levey, T. L. Jang, and P. Russo, "Partial nephrectomy versus radical nephrectomy in patients with small renal tumors-is there a difference in mortality and cardiovascular outcomes?” The Journal of Urology, vol. 181, no. 1, pp. 55-62, 2009.

[4] L. Zini, P. Perrotte, U. Capitanio et al., "Radical versus partial nephrectomy: effect on overall and noncancer mortality," Cancer, vol. 115, no. 7, pp. 1465-1471, 2009.

[5] B. Ljungberg, N. C. Cowan, D. C. Hanbury et al., "EAU guidelines on renal cell carcinoma: the 2010 update," European Urology, vol. 58, no. 3, pp. 398-406, 2010.

[6] W. K. O. Lau, M. L. Blute, A. L. Weaver, V. E. Torres, and H. Zincke, "Matched comparison of radical nephrectomy vs nephron-sparing surgery in patients with unilateral renal cell carcinoma and a normal contralateral kidney," Mayo Clinic Proceedings, vol. 75, no. 12, pp. 1236-1242, 2000.

[7] J. McKiernan, R. Simmons, J. Katz, and P. Russo, "Natural history of chronic renal insufficiency after partial and radical nephrectomy," Urology, vol. 59, no. 6, pp. 816-820, 2002.

[8] A. S. Go, G. M. Chertow, D. Fan, C. E. McCulloch, and C.-Y. $\mathrm{Hsu}$, "Chronic kidney disease and the risks of death, cardiovascular events, and hospitalization," The New England Journal of Medicine, vol. 351, no. 13, pp. 1296-1305, 2004.

[9] L. J. Barlow, R. Korets, M. Laudano, M. Benson, and J. McKiernan, "Predicting renal functional outcomes after surgery for renal cortical tumours: a multifactorial analysis," BJU International, vol. 106, no. 4, pp. 489-492, 2010.

[10] M. Hartleb and K. Gutkowski, "Kidneys in chronic liver diseases," World Journal of Gastroenterology, vol. 18, no. 24, pp. 3035-3049, 2012.

[11] A. Sharma, M. J. Mucino, and C. Ronco, "Renal functional reserve and renal recovery after acute kidney injury," NephronClinical Practice, vol. 127, no. 1-4, pp. 94-100, 2014.

[12] A. Fergany, "Chronic renal insufficiency after partial nephrectomy for T1b tumors," Current Opinion in Urology, vol. 23, no. 5, pp. 394-398, 2013.

[13] P. Russo, "Functional preservation in patients with renal cortical tumors: the rationale for partial nephrectomy," Current Urology Reports, vol. 9, no. 1, pp. 15-21, 2008.

[14] A. Mukkamala, C. He, A. Z. Weizer et al., "Long-term renal functional outcomes of minimally invasive partial nephrectomy for renal cell carcinoma," Urologic Oncology, vol. 32, no. 8, pp. 1247-1251, 2014. 
[15] X. Rod, B. Peyronnet, T. Seisen et al., "Impact of ischaemia time on renal function after partial nephrectomy: a systematic review," BJU International, vol. 118, no. 5, pp. 692-705, 2016.

[16] S. L. Hofbauer, A. J. Pantuck, M. de Martino et al., “The preoperative prognostic nutritional index is an independent predictor of survival in patients with renal cell carcinoma," Urologic Oncology, vol. 33, no. 2, pp. 68.e1-68.e7, 2015.

[17] S. H. Kim, S. E. Lee, S. K. Hong et al., "Incidence and risk factors of chronic kidney disease in Korean patients with Tla renal cell carcinoma before and after radical or partial nephrectomy," Japanese Journal of Clinical Oncology, vol. 43, no. 12, pp. 12431248, 2013.

[18] S. Rajan, R. Babazade, S. R. Govindarajan et al., "Perioperative factors associated with acute kidney injury after partial nephrectomy," British Journal of Anaesthesia, vol. 116, no. 1, pp. 70-76, 2016.

[19] C. S. E. Kim, E. H. U. Bae, S. K. W. Ma, S.-S. Kweon, and S. W. A. Kim, "Impact of partial nephrectomy on kidney function in patients with renal cell carcinoma," BMC Nephrology, vol. 15, article 181, 2014.

[20] H. J. Kong, J. S. Park, D. Y. Kim, H. S. Shin, and H. J. Jung, "Renal function following curative surgery for renal cell carcinoma: who is at risk for renal insufficiency?" Korean Journal of Urology, vol. 54, no. 12, pp. 830-833, 2013.

[21] L. Li, W. L. Lau, C. M. Rhee et al., "Risk of chronic kidney disease after cancer nephrectomy," Nature Reviews: Nephrology, vol. 10, no. 3, pp. 135-145, 2014.

[22] P. Satasivam, F. Reeves, K. Rao et al., "Patients with medical risk factors for chronic kidney disease are at increased risk of renal impairment despite the use of nephron-sparing surgery," $B J U$ International, vol. 116, no. 4, pp. 590-595, 2015.

[23] C. Pipili and E. Cholongitas, "Renal dysfunction in patients with cirrhosis: where do we stand?" World Journal of Gastrointestinal Pharmacology and Therapeutics, vol. 5, no. 3, pp. 156-168, 2014.

[24] F. De Ritis, M. Coltorti, and G. Giusti, "An enzymic test for the diagnosis of viral hepatitis: the transaminase serum activities," Clinica Chimica Acta, vol. 369, no. 2, pp. 148-152, 2006.

[25] A. Bezan, E. Mrsic, D. Krieger et al., "The preoperative AST/ ALT (De Ritis) ratio represents a poor prognostic factor in a cohort of patients with nonmetastatic renal cell carcinoma," The Journal of Urology, vol. 194, no. 1, pp. 30-35, 2015.

[26] O. Warburg, "On respiratory impairment in cancer cells," Science, vol. 124, no. 3215, pp. 269-270, 1956.

[27] W. V. V. Greenhouse and A. L. Lehringer, "Occurrence of the malate-aspartate shuttle in various tumor types," Cancer Research, vol. 36, no. 4, pp. 1392-1396, 1976. 


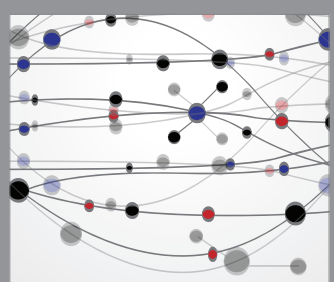

The Scientific World Journal
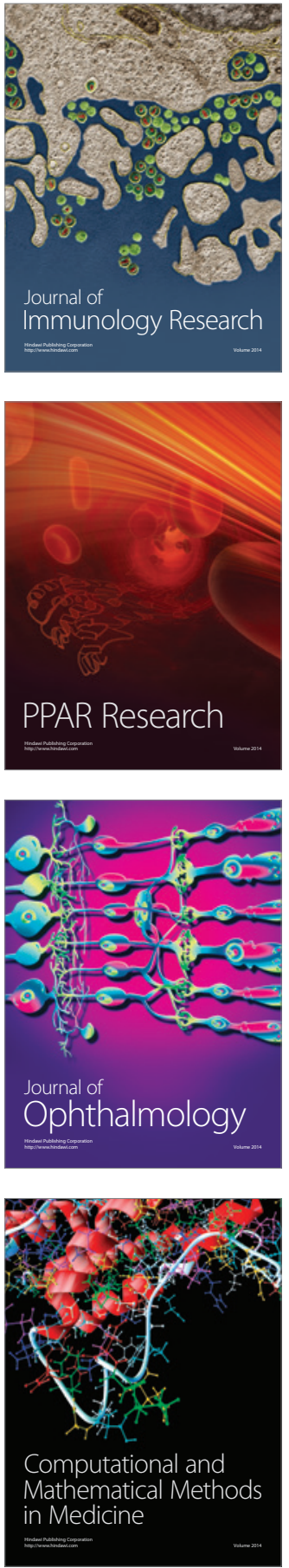

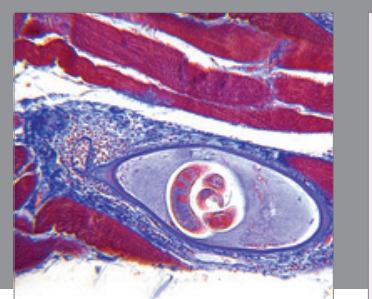

Gastroenterology Research and Practice

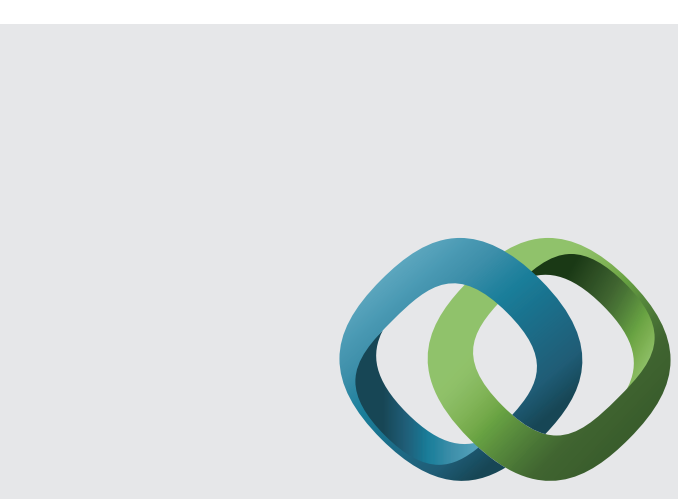

\section{Hindawi}

Submit your manuscripts at

http://www.hindawi.com
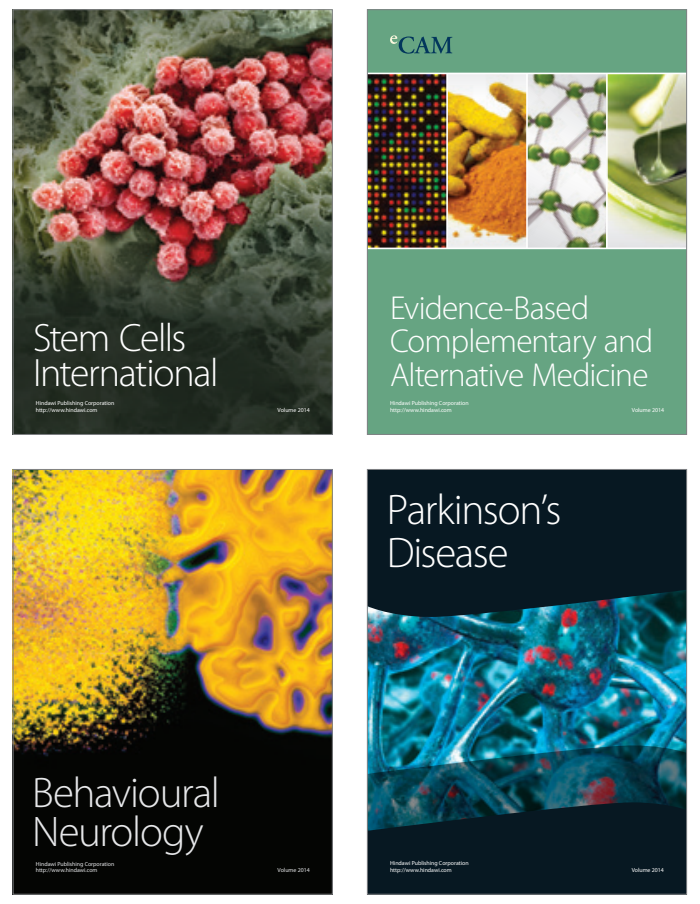
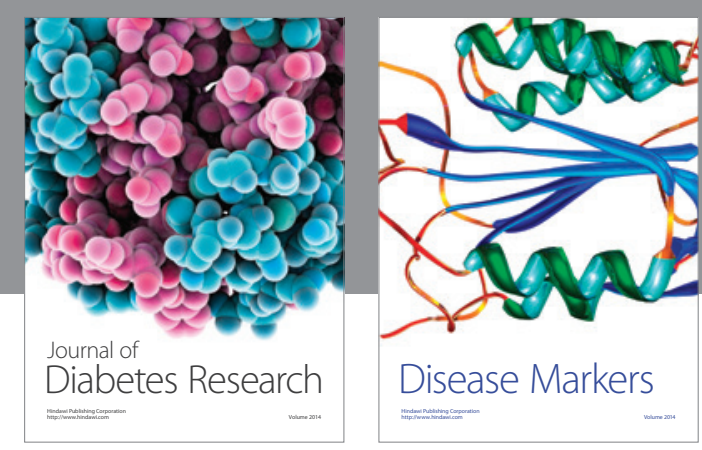

Disease Markers
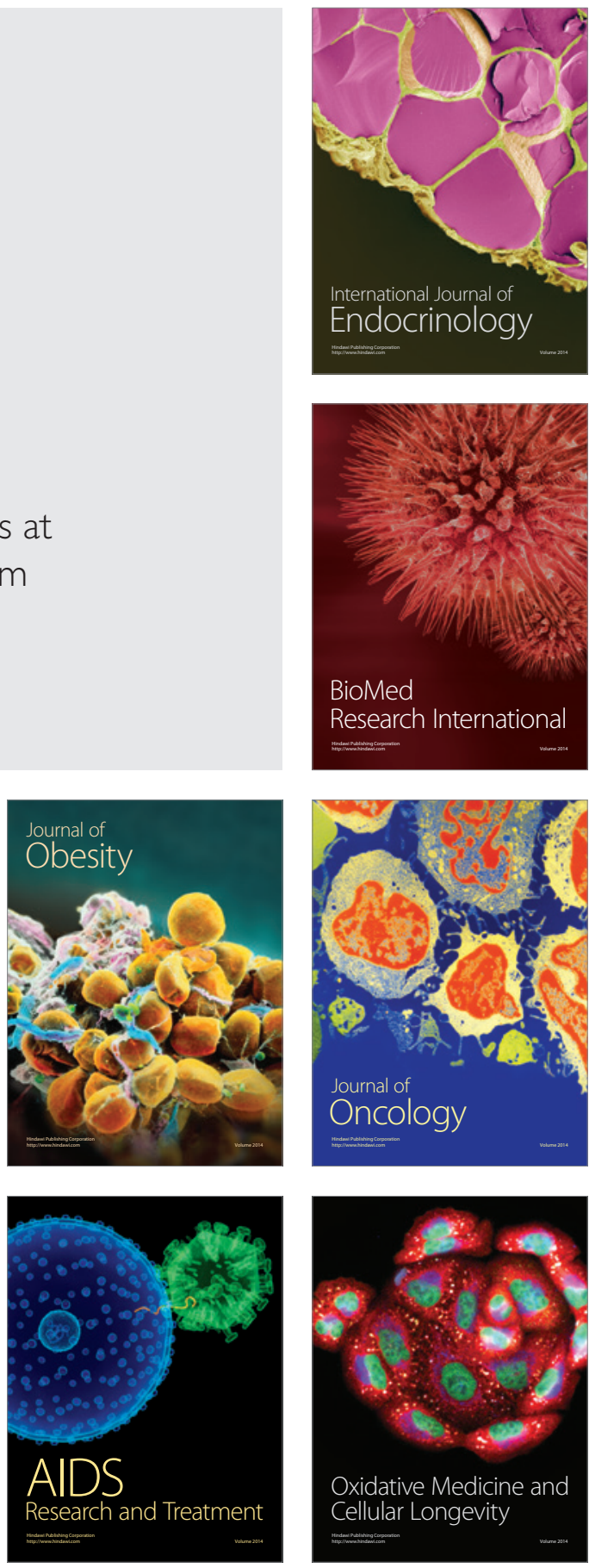